Kali A. Barrett

Damon C. Scales

\section{Considering the vulnerabilities of surrogate decision-makers when obtaining consent for critical care research}

Received: 12 October 2011

Accepted: 30 October 2011

Published online: 26 November 2011

(C) Copyright jointly held by Springer and ESICM 2011

This editorial refers to articles available at:

doi:10.1007/s00134-011-2411-1 and

doi:10.1007/s00134-011-2412-0.

\section{K. A. Barrett}

Department of Medicine, Faculty of Medicine,

University of Toronto, Toronto, Canada

D. C. Scales

Interdepartmental Division of Critical Care,

University of Toronto, Toronto, ON, Canada

D. C. Scales ( $)$

Department of Critical Care Medicine, Sunnybrook Health

Sciences Centre, 2075 Bayview Avenue, D108,

Toronto, ON M4N 3M5, Canada

e-mail: damon.scales@utoronto.ca

Tel.: +1-416-4805291

Fax: +1-416-4804999

Obtaining informed consent from critically ill patients to conduct research poses unique challenges. These patients are often incapable of providing informed first-person consent due to their underlying critical illness or the treatments they are receiving. In order to respect the ethical principle of autonomy, researchers commonly turn to substitute decision-makers (SDMs), for example, a spouse or other close relative, to obtain consent to conduct critical care research. However, these SDMs are often experiencing significant emotional strain and are also required to make decisions regarding medical treatment [1-3]. While most SDMs appear to want to participate in the research decision-making, this may add to the emotional burden of coping with a critically ill relative $[4,5]$.
In this issue of Intensive Care Medicine, two studies expand our understanding of the factors that influence consent rates in critical care research. Mehta and colleagues surveyed 95 SDMs who had previously been approached about enrolling their critically ill relative into a clinical trial to learn why they had agreed $(72 \%)$ or declined (28\%) to participate [6]. Those SDMs who had agreed to enroll their loved one most frequently cited that the research study might help others $(N=62$, $91 \%)$, that research is important for medical progress $(N=60,88 \%)$, that they trusted the medical team $(N=59,87 \%)$, and that they believed the patient would have agreed to participate $(N=48,71 \%)$. Conversely, the SDMs who had declined enrollment explained that they were too worried to consider a research study $(N=18,67 \%)$, they had discomfort with the patient receiving an experimental treatment $(N=10,37 \%)$, they were concerned the study might adversely affect medical care $(N=9,33 \%)$, or that the study carried more risk than benefit $(N=9,33 \%)$.

Also in this issue, Menon and colleagues [7] report the results of an observational study examining 271 actual consent encounters involving 45 different studies and a range of study designs targeting critically ill children. They also sought to identify factors that influenced consent rates, and they recorded the unsolicited reasons provided by SDMs for declining consent. They observed that consent rates were increased when a member of the patient's clinical team first introduced the research assistant. Interestingly, they did not observe differences in consent rates for observational studies versus clinical trials, but consent rates were lower after cardiac surgery. Menon's study also highlights the emotional burden experienced by SDMs; more than half $(53 \%)$ of the 45 nonconsenters who volunteered a reason for refusal blamed their anxiety level as the reason for declining to enroll their relative, followed by concerns over interventions associated with the study protocol (40\%). 
These two studies are important because they offer insights into SDMs' reasons for enrolling or not enrolling their critically ill loved one in ICU research. However, both studies also raise concerns about involving SDMs in consent to research decision-making because of the high level of anxiety they may be experiencing. It is worrisome that SDM emotional burden-and not research study factors-appeared to be an important determinant of nonenrollment in both studies. Substitute decision-making is predicated on estimating the wishes of the incapacitated patient, and not on the emotional status of the SDM. This raises the possibility that some potential research participants are being denied the opportunity to participate in research in which they might have wanted to be involved simply because their SDM is emotionally overwhelmed.

However, it is even more concerning that many of the SDMs-greater than $60 \%$ of those surveyed by Mehta and colleagues - believed their loved one might directly benefit from their involvement in research. Many $(39 \% ; 17 / 44)$ cited this as their major reason, highlighting the potential for therapeutic misconception. Therapeutic misconception refers to a lack of understanding by those being targeted by the research intervention that the defining purpose of clinical research is to produce generalizable knowledge, regardless of whether the subjects enrolled in the trial may potentially benefit from the intervention under study or from other aspects of the clinical trial [8]. These findings should raise concerns that the concept of clinical equipoise may not be adequately understood by some SDMs. Alternatively, these results could reflect the desperation many SDMs feel concerning their loved one's illness, and illustrate how any potential "new" treatment can elicit their hopes for a better outcome. Finally, some SDMs may feel obliged to enroll their loved one in a study to avoid disappointing the clinical team. Indeed, most $(83 \%)$ of the SDMs in the study from Menon and colleagues who declined to involve their child in clinical research volunteered a reason for this decision. One possibility for this high rate of unsolicited responses is that these SDMs felt compelled to provide explanations to assuage their feelings of guilt for declining involvement.
Both of these studies advance our understanding of the factors that influence research decision-making by SDMs of critically ill patients, and should help researchers improve their approach to seeking consent and increase participation rates. However, changes to research protocols and enrollment procedures aiming to increase enrollment might, in fact, pressure SDMs further and exploit their vulnerability. Requiring informed consent for research participation suggests that an important choice is being made, one that balances risks and benefits considering the best estimates of a patient's own wishes. Any attempts to achieve $100 \%$ consent rates should therefore be viewed skeptically, as these may risk coercing SDMs. Alternatively, for studies where the risk to patients is deemed to be minimal, for example, observational studies, waiving the need for informed consent may be the more appropriate strategy to avoid inducing additional anxiety and stress for SDMs.

The SDM model for informed consent is imperfect, but it is a solution that allows the patient's autonomy to be respected when they are incapable. The studies from Mehta's and Menon's groups are important additions because both highlight that SDMs may be making decisions about enrolling their critically ill relatives based on their emotional state, rather than based on estimations of their loved ones' wishes. Researchers should take this into consideration when preparing study materials and enrollment procedures, and seek strategies to minimize any additional stress or anxiety that research decisionmaking might entail for SDMs. Finally, Mehta's and Menon's results should serve to caution us that studies achieving exceptionally high consent rates may have unintentionally exploited SDMs' vulnerabilities or placed them at risk for therapeutic misconception. Researchers, editors, and peer reviewers must therefore not let their expectations for high recruitment rates inadvertently contribute to a culture of coercion.

Acknowledgments There was no funding source for this editorial. Damon C. Scales is supported by a New Investigator Award from the Canadian Institutes for Health Research.

\section{References}

1. Azoulay E, Pochard F, Kentish-Barnes $\mathrm{N}$, Chevret S, Aboab J, Adrie C, Annane D, Bleichner G, Bollaert PE, Darmon M, Fassier T, Galliot R, Garrouste-Orgeas $\mathrm{M}$, Goulenok C, Goldgran-Toledano D, Hayon J, Jourdain M, Kaidomar M, Laplace C, Larché J, Liotier J, Papazian L, Poisson C, Reignier J, Saidi F, Schlemmer B, FAMIREA Study Group (2005) Risk of post-traumatic stress symptoms in family members of intensive care unit patients. Am J Respir Crit Care Med 171:987-994
2. Pochard F, Darmon M, Fassier T, Bollaert PE, Cheval C, Coloigner M, Merouani A, Moulront S, Pigne E, Pingat J, Zahar JR, Schlemmer B, Azoulay E, French FAMIREA study group (2005) Symptoms of anxiety and depression in family members of intensive care unit patients before discharge or death. A prospective multicenter study. J Crit Care 20:90-96
3. Pochard F, Azoulay E, Chevret S, Lemaire F, Hubert P, Canoui P, Grassin M, Zittoun R, le Gall JR, Dhainaut JF, Schlemmer B, French FAMIREA Group (2001) Symptoms of anxiety and depression in family members of intensive care unit patients: ethical hypothesis regarding decision-making capacity. Crit Care Med 29:1893-1897 
4. Barrett KA, Athaide V, Smith OM, Bali A, McDonald E, Pinto R, Stevenson J, Go S, Elman J, Friedrich JO, Cook DJ, Scales DC, Ferguson ND (2009) Proxy Evaluations Rating Methods for Inclusion into Trials. Am J Respir Crit Care Med 179:A1573

5. Chenaud C, Merlani P, Verdon M, Ricou B (2009) Who should consent for research in adult intensive care? Preferences of patients and their relatives: a pilot study. J Med Ethics 35:709-712
6. Mehta S, Quittnat Pelletier F, Brown M, et al. (2011) Why substitute decision makers provide or decline consent for ICU research studies: a questionnaire study. Intensive Care Med. doi: 10.1007/s00134-011-2411-1

7. Menon K, Ward R, Gaboury I, et al. (2011) Factors affecting consent in pediatric critical care research. Intensive Care Med.doi: 10.1007/s00134-011-2412-0
8. Henderson GE, Churchill LR, Davis AM, Easter MM, Grady C, Joffe S, Kass N, King NM, Lidz CW, Miller FG, Nelson DK, Peppercorn J, Rothschild BB, Sankar P, Wilfond BS, Zimmer CR (2007) Clinical trials and medical care: defining the therapeutic misconception. PLoS Med 4:e324 